\title{
Identificación de metabolitos secundarios presentes en los frutos frescos de Cordia dentata Boraginaceae
}

\author{
Pamela Núñez ${ }^{1}$ \\ Luz Mejía, Leila Yacamán, Leda Padilla, Alejandra Coello, Janeli Ferrari, \\ Ramón Posadas ${ }^{2}$ \\ Ana Carolina Arévalo ${ }^{3}$
}

\section{RESUMEN}

En el presente estudio se dan a conocer las familias de metabolitos secundarios presentes en Cordia dentata Boraginaceae, conocida comúnmente como Tigüilote. La selección de $C$. dentata se llevó a cabo en la comunidad de Nacaome, a través de encuestas etnobotánicas aplicadas a los pobladores de la comunidad, quienes reportaron que utilizan los frutos de la plantas para tratar la tos y la fiebre.

Se realizó un protocolo de análisis para identificar las familias de metabolitos presentes en los frutos frescos de esta especie, con el objetivo de aportar datos químicos que avalen el uso tradicional que le da la comunidad a esta planta. A través de este estudio se demostró la presencia de taninos, alcaloides, cumarinas y heterósidos cianogenéticos en los frutos del tigüilote, metabolitos secundarios que podrían ser responsables de la actividad antipirética y antitusiva reportada para este taxón.

La presencia de heterósidos cianogenéticos podría justificar el uso para el tratamiento de la tos reportado por la comunidad y la presencia de cumarinas en el fruto podrían explicar el uso antipirético de esta planta, sin embargo, se recomienda continuar con los estudios farmacológicos para complementar el estudio químico realizado.

Se considera importante este tipo de estudios que avalen el uso tradicional de las plantas medicinales, ya que en los últimos años se ha evidenciado la necesidad de recurrir a la medicina alternativa para tratar las diferentes enfermedades, como consecuencia de los altos costos de los productos farmacéuticos.

\footnotetext{
${ }^{1}$ Instructora, Laboratorio del Departamento de Control Químico, Facultad de Química y Farmacia, UNAH.

${ }^{2}$ Estudiantes, Farmacognosia I, Departamento de Control Químico, Facultad de Química y Farmacia, UNAH

${ }^{3}$ Asesora, Ciencias Farmacéuticas, Departamento de Control Químico, Facultad de Química y Farmacia, UNAH. Contacto:ana.arevalo@unah.edu.hn
} 
Palabras clave: Cordia dentata, metabolitos secundarios, análisis fitoquímico.

\section{ABSTRACT}

In the present study we are identifying the secondary metabolite families present in Cordia dentata Borginacae, commonly known as Tigüilote.

C. dentata was selected by means of etnobotanic surveys taken among the residents of Nacome Valley, who reported the use of the fruit of the plant to treat cough and fever. An analysis protocol was performed to identify the metabolitic families present in the fresh fruits of this species for the purpose of providing chemical data that will demonstrate the value of the traditional use the community gives to this plant. By means of this study, the presence of tannins, alkaloides, coumarins and cianogenetic glycosides was demonstrated in the fruits of Tigüilote. These secondary metabolites that could be responsible for the antipuretic and antitusive activity reported for this taxon. We consider that this type of study, that validates the traditional uses of medicinal plants to be important, given that, in recent years, the need to recur to alternative treatments for different diseases has become evident due to the high cost of pharmaceutical products.

Key words: tigüilote, Cordia stellifera, secondary metabolite, phytochemical analysis.

\section{INTRODUCCIÓN}

Cordia dentata es una especie que crece en las zonas secas desde México hasta Panamá, en las Antillas, Colombia y Venezuela. Su principal uso es como madera para leña, pero en Honduras se reporta que en el valle de Comayagua se comercializa como madera para la fabricación de postes y cercas vivas (García, 2009). También es utilizado como forraje, ya que presenta valores aceptables de proteína cruda en las hojas (16-20 \%) y en menor proporción en los tallos (7\%). En comparación con otras especies de forraje, las hojas y los tallos presentan una baja digestibilidad por la presencia de taninos, aunque se reporta el uso de las hojas secas molidas y mezcladas con sorgo como suplemento proteico para gallinas, observándose un aumento en la producción diaria de huevos, sin embargo, no debe ser utilizado como dieta única (Flores, 1994).

C. dentata es un árbol pequeño, de 2-10 m de altura. Se caracteriza por su porte irregular, tronco corto, frecuentemente torcido y copa muy ramificada, presenta corteza gris o parda grisácea, muy fisurada. Hojas simples y alternas de 3 a $13.5 \mathrm{~cm}$ 
de largo, de 2 a $7 \mathrm{~cm}$ de ancho, elípticas ovadas o redondeadas, ápice agudo 0 redondeado; contienen pequeños dientes en el borde (de ahí el nombre científico dentata). Las flores son de amarillas pálidas a blancas, muy vistosas, producen néctar y son polinizadas por abejas y otros insectos; inflorescencia en cimas paniculadas, hasta de $20 \mathrm{~cm}$ de ancho, con muchas flores. El fruto es ovoide, de $1 \mathrm{a}$ $1.5 \mathrm{~cm}$ de largo, de $0.6 \mathrm{~cm}$ de diámetro, blanco y casi transparente cuando está maduro; es consumido por aves, monos, murciélagos e iguanas, atraídas por la pulpa jugosa y muy dulce (Medina, 1994).

Se reporta que las hojas son utilizadas en medicina tradicional como emolientes y las flores para la tos y como sudorífico. Los frutos de color blanco son dulces y comestibles, con un jugo mucilaginoso que se emplea como pegamento, se utiliza como repelente y para el tratamiento del sarampión (Zamora, 1999). Según la literatura consultada, se tienen reportes sobre el uso etnobotánico de las flores y las hojas para tratar la tos, pero no del fruto; sin embargo, en la comunidad de Nacaome se reporta el uso de los frutos frescos como tratamiento para la tos y la fiebre.

El presente trabajo tiene como objetivo validar científicamente algunos de los usos populares que se le dan al fruto fresco de Cordia dentata, así como contribuir al conocimiento de su composición química mediante el análisis fitoquímico de la especie. A través de este estudio se demostró la presencia de taninos, alcaloides, cumarinas y heterósidos cianogenéticos en los frutos del tigüilote, metabolitos secundarios que podrían ser responsables de la actividad antipirética y antitusiva reportada para este taxón.

\section{MÉTODOLOGÍA}

\section{Diseño}

Estudio descriptivo y transversal para determinar los metabolitos secundario presentes en Cordia dentata Boraginaceae (ver figura 1).

\section{Población}

Se seleccionó la planta conocida comúnmente como tigüilote, con nombre científico Cordia dentata Boraginaceae, a través de encuestas etnobotánicas realizadas en la comunidad de Nacaome, utilizando como guía un cuestionario estructurado. La comunidad reportó el uso del fruto del tigüilote para tratar la fiebre y la tos, en forma de infusión (ver figura 2 y 3 ). 


\section{Entorno}

Las encuestas etnobotánicas, recolección del material vegetal y la muestra de herbario se realizaron en octubre de 2013 , en la ciudad de Nacaome, municipio de Nacaome, departamento de Valle (ver figura4).

\section{Intervenciones}

1. Material vegetal: la identificación taxonómica de $C$. dentata se realizó en el herbario Cyril Hardy Nelson Sutherland T por la Dra. Lilian Ferrufino. Se dejó como respaldo un ejemplar en el herbario de la Facultad de Ciencias Químicas y Farmacia de la UNAH (ver figura 5).

2. Procesamiento del material vegetal: los frutos frescos de tigüilote se recolectaron en octubre de 2013, por la mañana, a una temperatura promedio de 33 grados centígrados en la ciudad de Nacaome. Se lavaron para eliminar materia extraña en el lugar de recolección, posteriormente se llevaron al Laboratorio de Farmacognosia I, en donde se refrigeraron para su conservación. En este Laboratorio los frutos se fragmentaron de forma manual y se sometieron a extracción por maceración estática, utilizando etanol $95 \%$ como solvente de extracción para realizar las diferentes pruebas de identificación de metabolitos secundarios.

3. Análisis fitoquímico: utilizando el material vegetal fresco se realizaron las pruebas de borntrager para identificar antraquinonas y la prueba de grignard para la identificación de heterosidos cianogenéticos.

4. A partir del extracto etanólico se realizó la identificación de alcaloides usando los reactivos de dragendorff, wagner, hager, silicotungstico, bouchardat. La presencia de taninos se evaluó con hidróxido de sodio más gelatina $1 \%$, cloruro férrico $1 \%$, formaldehido más ácido clorhídrico concentrado y ácido clorhídrico concentrado más calor. Las cumarinas se identifican usando hidróxido de sodio $10 \%$ y luz U.V. como revelador. Las pruebas de presencia/ausencia de flavonoides se realizaron con cloruro férrico, nitrato de plata, cloruro de aluminio y vapores de amoniaco, prueba de shinoda, álcalis, ácido sulfúrico y ácido sulfúrico más ácido bórico. Para la identificación de heterósidos cardiotónicos se realizaron las pruebas de baljet, libermann- bauchatd, salkowski (Solís, 2003). 


\section{RESULTADOS}

Los resultados obtenidos del estudio fitoquímico realizado en los frutos frescos de $C$. dentata se muestran en la tabla 1, con la determinación de metabolitos secundarios de C. dentata.

Tabla 1. Determinación de metabolitos secundarios de C. dentata

\begin{tabular}{|c|c|c|c|}
\hline $\begin{array}{l}\text { Metabolito } \\
\text { secundario }\end{array}$ & Prueba & Resultado & $\begin{array}{l}\text { Observación } \\
\text { por presencia }\end{array}$ \\
\hline Antraquinonas & Reacción de borntrager & - & Capa acuosa, rosa o rojo \\
\hline Cumarinas & $\begin{array}{l}\text { Hidróxido de sodio } 10 \\
\% \text { / luz U.V. }\end{array}$ & + & Luz U.V. \\
\hline $\begin{array}{l}\text { Heterósidos } \\
\text { Cianogenéticos }\end{array}$ & Grignard & + & Color rojo ladrillo \\
\hline Alcaloides & $\begin{array}{l}\text { Mayer } \\
\text { Dragendorff } \\
\text { Wagner } \\
\text { Hager } \\
\text { Silicotungstico } \\
\text { Bouchardat }\end{array}$ & $\begin{array}{l}+ \\
+ \\
+ \\
+ \\
+ \\
+\end{array}$ & $\begin{array}{l}\text { Precipitado color crema } \\
\text { Precipitado color anaranjado } \\
\text { Precipitado color café } \\
\text { Precipitado color amarillo } \\
\text { Precipitado color crema } \\
\text { Precipitado color crema }\end{array}$ \\
\hline Taninos & $\begin{array}{l}\text { Hidróxido de sodio + } \\
\text { gelatina1\% } \\
\text { Cloruro férrico } 1 \% \\
\text { Formaldehido + ácido } \\
\text { clorhídrico concentrado } \\
\text { Ácido clorhídrico } \\
\text { concentrado + calor }\end{array}$ & $\begin{array}{l}+ \\
- \\
+\end{array}$ & $\begin{array}{l}\text { Precipitado color crema } \\
\text { Precipitado color negro-azul } \\
\text { Precipitado rojizo } \\
\text { Precipitado color rojizo }\end{array}$ \\
\hline Flavonoides & $\begin{array}{l}\text { Shinoda } \\
\text { Álcalis } \\
\text { Ácido sulfúrico } \\
\text { Ácido sulfúrico y ácido } \\
\text { Bórico } \\
\text { Cloruro férrico } \\
\text { Nitrato de plata } \\
\text { Cloruro de aluminio } \\
\text { Vapores de amoniaco }\end{array}$ & $\begin{array}{l}- \\
- \\
-\end{array}$ & $\begin{array}{l}\text { Color rojo } \\
\text { Color amarillo } \\
\text { Color amarillo intenso } \\
\text { Fluorescencia verde } \\
\text { Color verde } \\
\text { Color café } \\
\text { Color amarillo } \\
\text { Color amarillo }\end{array}$ \\
\hline $\begin{array}{l}\text { Heterósidos } \\
\text { cardiotónicos }\end{array}$ & $\begin{array}{l}\text { Baljet A/B } \\
\text { Libermann- bauchatd } \\
\text { Salkowski }\end{array}$ & - & $\begin{array}{l}\text { Color amarillo } \\
\text { Color verde, azul, rojo } \\
\text { Color anaranjado/rojizo }\end{array}$ \\
\hline
\end{tabular}




\section{DISCUSIÓN}

Se identificó la presencia de alcaloides y taninos en los frutos frescos de $C$. dentata y metabolitos secundarios, previamente reportados en las hojas de la planta; García y colaboradores, en 2009, reportaron que estos compuestos por su baja concentración no deben ser considerados como factores antinutricionales cuando las hojas se usan como forraje para animales.

Además, se demostró la presencia de cumarinas en los frutos, principios activos que registró Villanueva, en el 2013, en el extracto etanolico de las hojas, en este mismo estudio también se reportó la presencia de triterpenos/esteroides y quinonas, metabolitos que resultaron negativos en el presente estudio.

En el presente análisis se identificó la presencia de heterósidos cianogenéticos, metabolitos secundarios con conocido efecto antitusivo, pero estos principios activos no se encuentran reportados en las hojas.

\section{CONCLUSIONES}

La comunidad de Nacaome reporta el uso del fruto fresco de $C$. dentata para tratar la tos y la fiebre. En el análisis fitoquímico se identificó la presencia de alcaloides, cumarinas, taninos y heterósidos cianogenéticos, aunque no se tienen reportes previos del uso de los frutos y no se encontraron en la literatura consultada análisis fitoquímicos sobre este órgano de la planta.

La presencia de heterósidos cianogenéticos podría justificar el uso para el tratamiento de la tos reportado por la comunidad y la presencia de cumarinas en el fruto podría justificar el uso antipirético de esta planta, sin embargo, se recomienda continuar con los estudios farmacológicos para complementar el estudio químico realizado.

Esta es una especie con alto potencial económico que crece de forma espontánea y actualmente se está promoviendo el cultivo en fincas porque evita la erosión del suelo; también se pueden utilizar las hojas y tallos como forraje y las hojas, flores y fruto en la medicina tradicional, lo que representa una especie rica en principios activos; en consecuencia, se recomienda el diseño de una forma farmacéutica para incorporar el extracto. 


\section{AGRADECIMIENTO}

Ala comunidad de Nacaome por el apoyo en el desarrollo del presente estudio.

\section{BIBLIOGRAFÍA}

Flores Ruano, O. I. (1994). Caracterización y evaluación de follajes arbóreos para la alimentación de rumiantes en el departamento de Chiquimula, Guatemala. En Benavides, J.E. Árboles y arbustos forrajeros en América Central. Costa Rica: CATIE.

García, A.; Abadía, B.; Barahona, R.; Sánchez, S. (2009). Caracterización fitoquímica de factores Antinutricionales en las hojas de uvito (Cordia dentata Poir). Revista MVZ Córdoba, 14(1).1611-1623, Medellín.

Medina, J.M.; Rouyer, B.; Tejada, M.; Layas, M.; Borion, B. (1994).Evaluación preliminar de la producción de biomasa de especies leñosas bajo crecimiento natural en la zona sur de Honduras. En Benavides, J.E. Árboles y arbustos forrajeros en América Central. Costa Rica: CATIE.

Solís, P.N.; Guerrero, N.; Gattuso, S.; Cáceres, A. (2003). Manual de caracterización y análisis de drogas vegetales y productos fitoterapéuticos. Panamá: Proyecto Desarrollo de Tecnología de Cultivo de Plantas Medicinales y Producción de Fitoterápicos.

Villanueva, C.; Castillo, F.; Estrada, H. (2013). Preliminary phytochemical screening of promising plant species of the Colombian Atlantic coast. Rev. Cubana Plant Med, 18(4), 619-631. Cuba.

Zamora, N.; González J. y Poveda L. J. (1999). Árboles y arbustos del bosque seco de Costa Rica. Costa Rica: Instituto Nacional de Biodiversidad.

Figura 1. Frutos frescos de Cordia dentata Boraginaceae

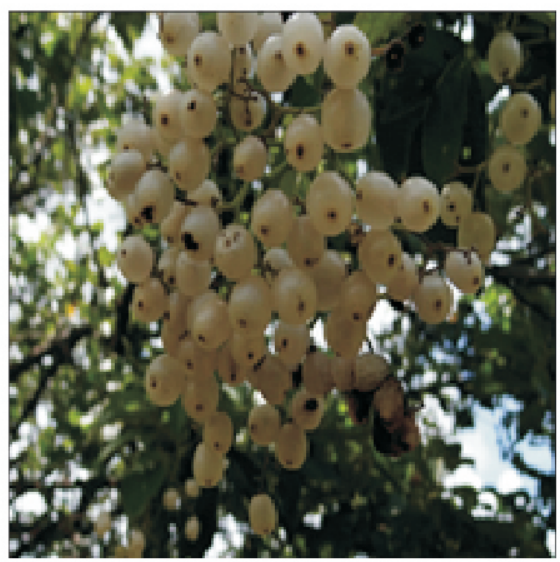


Figura 2. Encuesta etnobotánica

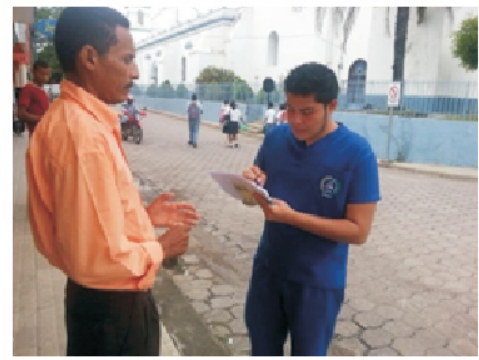

Figura 3. Encuesta etnobótanica

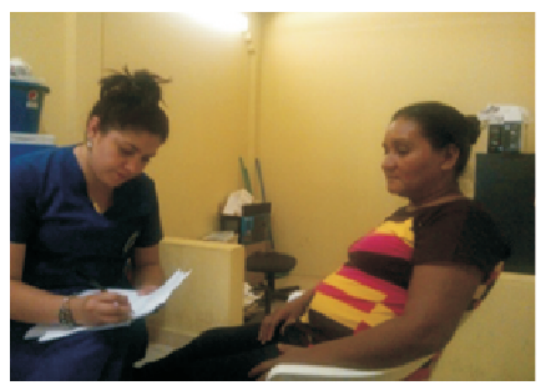

Figura 4. Recolección del material vegetal y la muestra de herbario

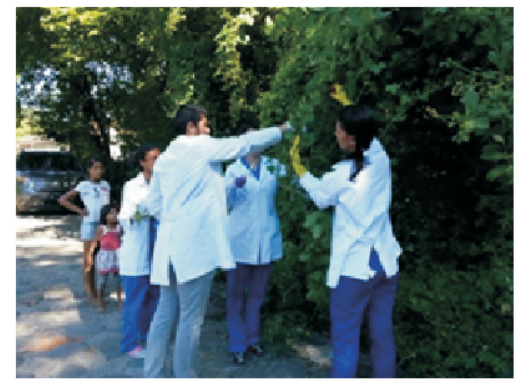

Figura 5. Recolección del material vegetal y la muestra de herbario

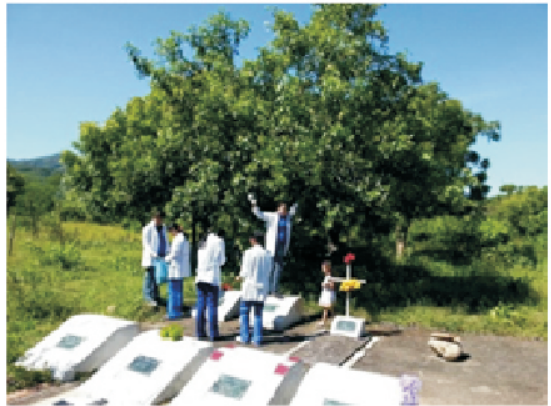

